\title{
FORMALISMO Y METAFICCIÓN: WILLIAM GASS Y LA GENERACIÓN DE ESCRITORES AUTORREFLEXIVOS NORTEAMERICANOS ${ }^{1}$
}

\author{
Belén Piqueras Cabrerizo ${ }^{2}$
}

\begin{abstract}
Resumen: William Gass y un grupo de escritores norteamericanos afines que desarrollaron el grueso de su obra en los años 1960 y 1970, practicaron lo que dio en llamarse novela autorreflexiva o metaficción, usándola como plataforma donde reflexionar con un escepticismo radical sobre la realidad percibida desde la ficción o la autoridad narrativa, llegando a cuestionarse la noción misma de literatura. Dominados por un espíritu disidente, estos escritores desafiaron el control de las autoridades políticas y literarias mediante la denuncia del carácter artificial e ilusorio del lenguaje de uso social y de la falacia que éste origina en el ámbito de la narrativa.

Palabras clave: Formalismo, metaficción, metáfora, modernismo, postmodernismo.

Formalism and Metafiction: William Gass and the Generation of Self-reflective American Writers

Abstract: William Gass and a group of congenial American writers that developed the bulk of their work during the 1960s and 70s produced what was defined as self-reflexive novel or metafiction, making of it a showcase of their sceptical positions concerning fiction and narrative authority as a means of conveying reality, and getting even to question the very notion of literature. Dissenting and nonconformist, these writers boldly confronted the control of political and literary authorities, appealing to the artificial and delusive nature of language's social function and to the fallacy it constitutes in the realm of fiction.
\end{abstract}

Keywords: Formalism, metafiction, metaphor, modernism, postmodernism.

La novela y la poesía estadounidenses de los años cincuenta fueron, en general, convencionales y conformistas, no dando apenas muestras de impulsos experimentales como había sucedido en generaciones anteriores. La nota discordante de esta época la marcó la Generación Beat ${ }^{3}$, que fue, además de un modo de protesta social, una estridencia literaria de marcado carácter político que se oponía a la imagen del poeta como hombre de letras, celebrando las experiencias más extremas como forma espontánea de inspiración. Los

\footnotetext{
$1 \quad$ Fecha de recepción: febrero 2009.

Fecha de aceptación y versión definitiva: abril 2009.

2 Profesora Asociada, Departamento de Filología Inglesa, Universidad Autónoma de Madrid; $\square$ belen. piqueras@uam.es.

3 'In the beginning, in New York in the mid-1949's was a group which apparently included Kerouac, Allen Ginsberg, William S. Burroughs, Clellon Holmes, Carl Solomon, and Neal Cassady, the Dean Moriarty of On the Road. They were haunted by their inability to believe in anything, convinced that this faithlessness was unbearable, and driven by the tension arising from their conflicting views to a craving for excess. In 1948, Kerouac said to Holmes of this group and their situation: 'You know, this is really a beat generation."' (Webb 1974: 121)
} 
años cincuenta en poesía se caracterizaron por la existencia de una obra alternativa a la de los poetas que se veían a sí mismos como "mayores" -Lowell, Berryman, Jarrell, Dylan Thomas, etc.- elaborada, junto con los Beat, por grupos disidentes como los Black Mountain, los confesionales y los poetas de Nueva York, enemigos de la rigidez formalista que marcaba la Nueva Crítica.

Para muchos, la aparición en 1959 de The Naked Lunch, de William Burroughs, marca un cambio de orientación en el impulso creador de la época, no tanto por el espíritu de lo absurdo que exhibe, y que ya era en mayor o menor grado habitual en la narrativa estadounidense del decenio de 1950, sino por ser pionera en un método que se recrea en la "ficcionalidad" de la ficción, exponiendo de un modo lúdico las claves y los condicionantes formales del propio texto ${ }^{4}$; The Naked Lunch será la primera en publicarse de un conjunto de novelas que de un modo u otro "hablan" de sí mismas y del lenguaje que las constituye, tratando de llamar la atención sobre su condición de artefactos verbales. Esta nueva manera de entender la narrativa va a caracterizar gran parte de la literatura americana de los años sesenta y setenta y, aunque especialmente en la primera década de esta etapa no se abandonan la historia y la realidad como puntos de referencia, la función mimética va a ir progresivamente debilitándose en este tipo de creación literaria.

En su exploración de nuevas formas expresivas y en su intento de desestabilizar el discurso literario público, este periodo exhibe tantas variaciones como escritores forman parte de lo que ha dado en llamarse postmodernidad; pero las inquietudes de los escritores que practican la ficción autorreflexiva o metaficción -término éste último acuñado por William Gass- no son nuevas; experimentos de este tipo habían aparecido ya de forma explícita en Tristam Shandy (1759-1767) de Lawrence Sterne o en Through the Looking Glass (1871) de Charles Dodgson (Lewis Carroll), e incluso hay vestigios de técnicas autorreflexivas en Cervantes, Rabelais y Diderot ${ }^{5}$.

La metaficción, si tiene un espíritu común, es un espíritu disidente, pues la desestabilización semántica que produce este modo de escritura se deriva, en la mayoría de los autores que la practican, del rechazo a la rigidez y parcialidad que a su modo de ver caracterizan al realismo y al naturalismo; según ellos, este tipo de manifestaciones estéticas ya no se adaptan a la sociedad contemporánea y sus modelos obsoletos parecen incapaces de expresar las inquietudes del hombre moderno, que se cuestiona ahora más que nunca la relación entre su mundo y los lenguajes que lo articulan. Estas novelas reflexionan en su mayoría sobre cuestiones verbales, a su vez con un lenguaje contradictorio y a menudo disparatado que rezuma una palpable tensión: la que se origina en la disyuntiva de querer expresar con palabras a la vez que se trata de relegar el carácter inmediatamente utilitario o funcional de las mismas.

\footnotetext{
4 La ficcionalidad y autoreferencialidad de esta época constituyen, asimismo, consecuencias o epígonos del horror de la realidad vivida y muestras de la desconfianza o sospecha que se cierne sobre el lenguaje y sobre la imaginación liberal.

5 Para el escritor Carlos Fuentes, El Quijote de Cervantes supone una ruptura y una renovación del género novelístico. Con esta obra, la novela se convierte en un proyecto crítico que va a definir una tradición, una forma de hacer literatura hasta la actualidad. Así lo afirma Martha Elena Vernier en su ensayo "Escritura lateral: Ensayos de Carlos Fuentes", y allí recoge las siguientes palabras del propio Fuentes: "Cervantes es el primer novelista que radica su crítica de la creación dentro de las páginas de su propia creación, Don Quijote. Y esa crítica de la creación es una crítica del acto mismo de la lectura." (334)
} 
Si bien es cierto que la tradición realista ha coexistido siempre con todo tipo de experimentalismo literario, buscando formas convencionales de generar una sensación de orden social o histórico, es también indiscutible que, paralelamente, la postmodernidad es heredera directa de la modernidad; entendida bien como continuación o como rechazo de las inquietudes poéticas de las primeras décadas del siglo XX -cuestión planteada por algunos críticos de un modo demasiado maniqueo- la novela autorreflexiva cuestiona, pero también desarrolla, muchos de los principios artísticos que fueron pilares de la estética moderna. En líneas generales, ésta rompe con el tipo de representación metafórica y simbólica de la realidad que proponían los autores clásicos de la modernidad, y se rebela a su marcado carácter programático; pero, por otro lado, concibe nuevas formas de llevar a la práctica la sensibilidad que aquéllos ya mostraron por la palabra y por las cualidades más materiales y convencionalmente menos expresivas del medio verbal y de la que fue máximo exponente Gertrude Stein.

Si se buscan las causas culturales que condujeron a esta forma de narrativa contemporánea, hay que prestar especial atención a dos factores: por un lado, al socio-político, constituido por una generación desengañada de los discursos oficiales que se vieron desacreditados por las manipulaciones y mentiras de la administración Nixon, la guerra del Vietnam y el Watergate. Por otro, a una tradición cultural marcada ya de un modo irreversible por los planteamientos formales de la Nueva Crítica y por la reconceptualización del funcionamiento del signo en el ámbito de las artes. La línea entre la realidad y la ficción ha sido borrada, y los mecanismos de la representación se someten con más energía que nunca a un análisis crítico implacable por parte de escritores y filósofos.

Este es el contexto al que pertenece la obra de William Gass, cuya producción se relaciona habitualmente con la de John Barth, Robert Coover, Donald Barthelme, Thomas Pynchon, Ronald Sukenick, Philip Roth, Ishmael Reed, Jerzy Kosinski, Richard Brautigan, William Burroughs, John Hawkes, Kurt Vonnegut, Jr., entre otros. Ellos constituyeron una primera generación de escritores autorreflexivos que, aunque con nuevas estrategias formales, en general no abandonaron el modo narrativo en sus novelas.

Las obras de la mayoría de los escritores de esta primera generación autorreflexiva tratan de desmitificar la historia y el lenguaje americanos, y se implican en asuntos del pasado más o menos reciente de su país, ofreciendo una visión paródica e irónica de los mismos, o exhiben los convencionalismos de formas literarias previas. Algunos de estos autores se dedican a desafiar los grandes mitos contemporáneos, otros exploran las circunstancias de la existencia en un universo amenazador, otros meditan sobre las redes verbales que estructuran el mundo; todo ello ocurre dentro del marco de lo cómico y lo absurdo, mostrando unos personajes sin sustancia psicológica que contribuyen a configurar el carácter lúdico de una narrativa que juega de forma creativa con las convenciones literarias ${ }^{6}$.

\footnotetext{
6 En cuanto a la noción de personaje plano y sin sustancia psicológica, habría que remitirse a Michel Foucault, cuyos presupuestos filosóficos en torno a lo que ha dado en llamarse la "crisis del sujeto" han condicionado en gran medida la literatura del siglo XX. Entre los diversos ámbitos de esta crisis se destacará aquí el de la crisis del sujeto discursivo, por ser el que afecta en mayor grado a las cuestiones literarias que aquí se debaten.

Por un lado, Foucault entiende que los orígenes de la ficción se encuentran en el propio acto de la escritura. La práctica discursiva no es, según él, una operación expresiva mediante la cual un individuo formula una idea o un deseo, por lo tanto, no hay un sujeto que deja su huella en el discurso; el autor pasa a ser una función para Foucault. Asimismo, los “objetos" del discurso no deben considerarse como entes naturales. Los Discursos de-
} 
La narrativa de Gass elude directamente -que no indirectamente- la temática social y política que todavía muestran, como se acaba de afirmar, muchas de las obras de la primera oleada metaficcional, pero comparte con ellas el carácter básicamente lúdico -entendiendo lo lúdico en este contexto siempre como subversión- que las caracteriza de un modo genérico. Sin embargo, y a excepción de su primera novela, Omensetter's Luck, enmarcada en la tradición americana y con un componente argumental que no se repetirá en ninguna obra posterior del autor, la producción de Gass adquiere más significado dentro de una segunda fase de obras de metaficción que aparecieron en los años setenta, mucho más comprometidas con el proyecto común de dinamitar desde dentro de la obra el carácter representacional y descriptivo del lenguaje, poniendo en práctica muchas de las ideas de autores modernos tardíos como Nabokov, Borges o Beckett.

El rechazo a las formas tradicionales de narrar es ahora definitivo; estos autores escenifican una traición estridente a la mimesis, y ofrecen un nuevo tipo de texto que está completamente volcado en la exhibición llamativa y provocadora de sus procesos verbales. Son piezas de lectura incómoda ${ }^{7}$, dado que en su mayoría recurren a la dislocación de la prosa, a la fragmentación de la narrativa, a juegos visuales y tipográficos, a la incongruencia y a la contradicción dentro del propio texto; otras ofrecen verdaderos collages hechos con múltiples texturas "residuales" de una civilización verbalmente determinada; por último, las hay que incorporan su propia teoría, convirtiéndose en una especie de texto híbrido entre la ficción y la crítica literaria.

En realidad, esta división entre primera y segunda fase es algo forzada, puesto que muchos autores están a caballo entre ambas, generando alternativamente obras de mayor componente temático o mayor compromiso autorreflexivo, como es el caso de Coover, Barthelme o Sukenick, aunque algunos escritores pertenecen de un modo más explícito a la segunda tendencia, como Walter Abish, Steve Katz, Clarence Major o Gilbert Sorrentino, dadas sus estridencias metaficcionales.

La inclusión de Gass en este segundo grupo tiene que ver con su escepticismo a la hora de enfocar la problemática de la referencia verbal pues, aunque su proyecto se mueve en torno a la búsqueda tenaz de formas metafóricas que acerquen el lenguaje a la realidad, lo

\footnotetext{
ben ser entendidos como prácticas que obedecen a reglas determinadas, y será el propio juego de estas reglas el que unifique los enunciados, y no circunstancias ajenas a ellos. Pedro M. Hurtado Valero explica este asunto en un estudio sobre Foucault: "El discurso no es signo de una cosa que esté más allá de él y antes de él; por tanto, hay que dar de lado a las 'cosas', presuntamente anteriores al discurso para ceñirse exclusivamente" y Hurtado cita al Foucault de La arqueología del saber, “a 'la formación regular de los objetos que en él se dibujan' y 'las reglas que permiten formarlos como objetos de un discurso y constituyen así sus condiciones de aparición histórica"” (Hurtado 1994: 37).

Estas doctrinas de Foucault con respecto al discurso constituyen todo un precedente para escritores que son reacios a concebir sus personajes como objetos de estudio para la psicología tradicional, como si de seres humanos se tratase; los autores que, como Gass, entienden que todos los elementos de la obra, incluidos los personajes, adquieren sentido básicamente por ser parte integrante del entramado que constituye el texto, más que por relaciones de semejanza con circunstancias externas a él, encuentran un sólido fundamento filosófico en la obra de Foucault.

7 Esta incomodidad está relacionada con una postura hermenéutica de significación no cerrada que, especialmente a partir de los dictados de la Nueva Crítica, incorpora al lector a constituir el significado de la obra. La Nueva Crítica exigía más del lector, que debía convertirse en un participante activo a la hora de descodificar el texto, y dejar de ser un simple receptor pasivo del mismo.
} 
hace a sabiendas de la impracticabilidad de sus planteamientos. Gass se erige en portavoz de la estética anti-mimética, y él afirma con insistencia que sus intereses se dirigen a la elaboración de "objetos" artísticos únicos, no interpretables de forma unívoca por su semejanza o proximidad con el mundo; sin embargo, su poética propone nuevas formas de referencia intuitiva, jugando a resolver un problema de cuya irresolubilidad es consciente.

El espíritu del proyecto formalista está muy vinculado al Fiction Collective de Brooklyn, fundado en 1974 por un grupo de escritores, entre ellos Baumbach, Sukenick, Coover, Federman y Barthelme. Se trata de una empresa de edición cooperativa que pretendió ser una alternativa a las grandes editoriales cuyas miras exclusivamente comerciales dificultaban el acceso al mercado de obras experimentales de calidad como la mayoría de las producidas dentro del grupo - el propio Gass ha sido siempre consciente, y así lo reflejan ensayos como "The Writer and Politics: A Litany" o "The Shears of the Censor", dentro de su libro Tests of Time (2002) de las presiones y limitaciones que la sociedad de consumo y la dictadura del mercado ejercen sobre el escritor. Hasta 1988 lograron, a pesar de las innumerables dificultades, publicar cerca de cincuenta obras con este perfil, pero ello dañó notablemente la imagen y el futuro editorial de algunos de ellos, como Malamud, Roth, Brautigan y Coover. Según Ronald Sukenick, dos fueron los problemas fundamentales con que se topó este grupo de escritores: por un lado, con el hecho de que tan sólo un reducido número de librerías estaban dispuestas a ofrecer publicaciones literarias que no procedieran de la gran industria editorial; por otro, la escasez de información que acerca del mundo literario circula fuera de estos gigantes editoriales. Ambos problemas tienen que ver, afirma Sukenick, con el apoyo económico que dichas compañías reciben del gobierno y de diferentes fundaciones a fin de mantener una red de distribución que por sí misma ya sería suficientemente sólida (Sukenick 1985: 62-4).

Pero el Fiction Collective es mucho más que una alternativa editorial, y así lo entiende Sukenick cuando afirma:

When the Fiction Collective decided, in its own small way, to 'seize the means of production and distribution,' it was an assertion of power, a symbolic act of rebellion, a move toward realization of the ideal of writer as free spirit, demiurge, critic of the zeitgeist. It also represented a psychological liberation in a culture that tends to infantilize its artists: you must be helpless, irresponsible, and self-destructive -or else. Or else what? Or else you're not a real artist. But there is one other factor. Publishers are not just ordinary bosses; they are word bosses. They have enormous power to decide what language is good and what language is bad, and to back up those decisions by saturating the market with millions of copies of their books. Language control is mind control. (...). It is extremely odd that the Fiction Collective, with its tiny list of six books a year in face of the thousands and thousands pumped out by the industry, should have caused such a fuss, that it still, after five years, annoys the publishers and arouses controversy. It must be at least partly because if you think about the Collective at all, you have to think about these issues. (Sukenick 1985: 59)

El proyecto del Fiction Collective se corresponde, pues, con una actitud tanto estética como política, y el tipo de reivindicación que sus miembros llevan a cabo se refleja en sus obras, que apuntan, en el sentido más amplio, hacia una liberación del lenguaje creativo 
-el control de la mente a través del lenguaje, como denuncia Sukenick arriba, es el argumento que sustenta la gran mayoría de sus denuncias. Este grupo de escritores, que se autodenominan formalistas, elabora un tipo de ficción que, lejos de ser verosímil, utiliza diversos "motivos" conceptuales o estructurales para establecer un orden y un sistema de relaciones nuevas dentro de la obra, dando vida a un "ente" textual equilibrado y coherente en su propia ontología.

La realidad que estos autores presentan pretende liberarse del componente semiótico habitual de las palabras para elaborar un nuevo dominio de referencia que depende esencialmente del lenguaje que lo crea -lógicamente, este nuevo dominio es asimismo semiótico, aunque responde a nuevas reglas. Ellos conciben así sistemas formales que tratan de poner en evidencia la arbitrariedad del lenguaje convencional. De este modo, para Sukenick, en el mundo del postrealismo, todos los absolutos del realismo se hacen problemáticos, y así lo plantea en su libro The Death of the Novel and Other Stories (1969), donde reflexiona con un escepticismo radical sobre la "realidad" percibida desde la ficción o la autoridad narrativa, llegando a cuestionarse la propia noción de literatura. Sobre esta obra afirma el propio autor:

In The Death of the Novel and Other Stories, I attempted, with intentional naivete, a new realism by taking imprints of 'reality' with a tape recorder with varying combinations of such foreground/background relations in order to move beyond the impasse of narrative authority implied by the general increase in our consciousness of linguistic and narrative limitations. (Sukenick 1985: 78)

Esta voz disidente refleja en gran medida el espíritu que predomina entre los escritores americanos de los años sesenta y setenta, muchos de los cuales pretenden desafiar el control y las imposiciones de las autoridades políticas y literarias mediante la denuncia del carácter artificial e ilusorio del lenguaje de uso social y de la falacia que éste origina en el ámbito de la ficción.

Entendido como instrumento de la comunicación, el lenguaje permite la transmisión de información de unos hablantes a otros. Sin embargo, este mito culturalmente asimilado conlleva varios errores; por un lado, ignora que tal información ha de ser traducida en los códigos verbales, lo que necesariamente desvirtúa su objetividad; por otro, y más importante, implica una concepción errónea del vehículo expresivo, atribuyéndole una neutralidad, estabilidad e inmanencia que las lenguas en uso no tienen. Charles Bernstein ha tratado de ilustrar este modo erróneo de entender la comunicación mediante la que él ha denominado teoría del tubo según la cual el hablante concibe la transmisión de sus ideas mediante el lenguaje como si lo hiciera a través de un tubo o manguera a cuyo extremo se encuentra su interlocutor, que las recibe de un modo riguroso y preciso (Bernstein 1986).

Muchos escritores contemporáneos, William Gass y el grupo del Fiction Collective entre otros, se rebelan contra esta concepción del lenguaje como vehículo, así como contra la idea de la significación "transparente" o inmediata que concede un valor didáctico y moral a la literatura; aunque es así como la conciben la mayoría de los hablantes, esto constituye un error que tiene consecuencias epistemológicas, estéticas y políticas. Por ello hacen del medio verbal el objetivo último de su creación, experimentando con estrategias diversas 
para llamar la atención sobre sus propias cualidades expresivas, y buscando nuevas maneras de hacer significar a la obra. Pretenden así huir de los convencionalismos del lenguaje cotidiano y del ámbito de lo colectivo, convirtiendo a las palabras en protagonistas de una manifestación artística singular. Desprovista de su valor mimético, educativo o moral -términos que pueden parecer antitéticos, pero que no lo son en realidad, pues, el que no tenga un valor "ejemplarizante" no significa que no sea educativa o "moral"- la novela puede ser casi cualquier cosa que uno desee, y este nuevo modo de concebir la narrativa suscitó en esta década de los setenta innumerables polémicas, en alguna de las cuales Gass participó activamente.

Este es el caso de su famoso debate con John Gardner en octubre de 1978 en la universidad de Cincinnati -reproducido en LeClair and McCaffery 1982-, y que, aunque falto de interés para algunos -Gass y Gardner eran buenos amigos, además de haber sido Gardner de gran ayuda para Gass en sus primeras publicaciones- sirvió para delimitar dos posturas rivales con respecto a la situación de la literatura en aquel periodo. Gardner, que acababa de publicar su obra On Moral Fiction (1977) mantenía, como el título de su libro indica, una postura tradicional ante la narrativa, mientras que Gass, alimentando con comentarios paralelos la ambigüedad que a veces destila su proyecto, se negaba a concederle a la literatura ningún "uso" que no fuera el del puro deleite estético. Así lo ha afirmado Gass reiteradamente, por ejemplo en una conversación a tres bandas con su amigo Stanley Elkin y el crítico Jeffrey L. Duncan: “(...) it again depends on whether or not you regard (that's why so much is assumed here) literature as fundamentally a kind of communication. If so, then the writer could make assertions that he regards as positive or negative. But I think that literature is not a form of communication" (Duncan 1976: 49).

La literatura que no comunica en el sentido estricto de la palabra, no es, sin embargo, necesariamente amoral, y es evidente que Gass mantenía esta postura mientras debatía con su amigo Gardner; en realidad, quizá no haya literatura mas "moral" que aquélla que aspira a añadir algo al mundo, como la de Gass.

Esta noción reduccionista con que Gass aborda el asunto de la comunicación en la cita anterior, que se asemeja al simplismo de la teoría de la manguera, no es aceptable por un lector contemporáneo consciente de que todo tipo de escritura, incluso la "no referencial" - puede que ésta aún más- constituye de un modo u otro una forma de comunicación. Sin embargo, todo lector familiarizado con el modo metafórico del discurso de Gass, que el autor no abandona ni siquiera en sus escritos de género ensayístico, sospecha de cualquier juicio de carácter objetivo o teórico que éste emita; sus afirmaciones están habitualmente rodeadas de un halo de ambigüedad que el uso mismo de la metáfora propicia, dentro siempre del juego contradictorio de afirmación-no afirmación que caracteriza la obra de Gass.

Lo que intenta impugnar Gass en la cita anterior es, precisamente, la simplicidad de la teoría de la manguera aplicada a la narrativa, es decir, se trata de un rechazo a toda posible noción "stendhaliana"-Stendhal definió la ficción como un espejo o ventana hacia la vida- de la novela. Gass entiende que este género ha causado un daño irreparable al arte de la escritura, pues ha inducido a una concepción errónea de la literatura que aún hoy en día es bastante generalizada (Gass 1989: 284-5). Puesto que el elemento que da cuerpo a la literatura es el mismo lenguaje que usa con fines comunicativos el sujeto social, en la ficción, éste crea la peligrosa sensación de que el personaje vive justo al otro lado de la página. Pero Gass quiere 
insistir en que los personajes y situaciones del texto no existen fuera del lenguaje, y de aquí el hecho de que el autor se identifique con el grupo de escritores que centran su interés en la elaboración de sistemas semánticos cuyo significado se construye con el propio texto y no en realidades ajenas a él.

Retomando de nuevo la noción de formalismo, conviene explicar que tanto para Gass como para los escritores metaficcionales de segunda generación con los que se le relaciona, el concepto tiene una acepción radicalmente diferente de la que tradicionalmente se le venía aplicando. Como afirma el propio Gass: "A lot of people understand a formalist simply to be someone who likes to practise the genres; particularly in poetry, a formalist is somebody who's gone back to strict verse forms, rhyme schemes and things of this sort. That's not what I mean by formalism" (Piqueras, Belén. "An Interview with William Gass". San Luis. 1995. Sin publicar). Y explica a continuación las nuevas consecuencias del término: "(...) And so, I'm a formalist in the sense that I would reject that, and I would concentrate on the way things are established in the text itself. In that sense, one wants to think of formalism in the sense of logic, mathematics, internal coherence, things of the sort, yet, I'm a formalist."

Aunque la noción de forma adquiere distintos matices dependiendo del escritor -más "dinámica" en el caso de Sukenick, por ejemplo, o más "estructural" en el caso de Gassqueda claro, pues, que la significación del texto concebida bajo estos criterios no depende tanto de un marco de referencia externa como de una serie de conexiones intratextuales que le dan un nuevo valor semántico. De este modo hay que interpretar la afirmación de William Gass de que el referente "no existe":

(...) The referent doesn't exist. (...) If I were trying to describe a thing in the world, the description would soon replace the thing, and it does all the time in a work of fiction. So then, the question is, you never did have a pen, what you have is the description of a pen, and the description has its own system, its own significance. And the pen, as a pen, is in no way phallic, but when I get it into a description, it can become. (Piqueras 1995)

El referente, como elemento del signo saussuriano que es, es un ente abstracto y, como tal, inaccesible; confundir el referente con la realidad supone un grave equívoco, y lo que Gass denuncia precisamente en la cita anterior es la distancia insalvable que hay entre el lenguaje y los objetos que denota. Ésta es, según él, la gran falacia de los sistemas de referencia convencional, deficiencia expresiva que el autor juega a solventar gracias a las virtudes tropológicas de la metáfora y a la condición esencialmente intuitiva de la metaficción.

Pero existen variaciones a la noción de referencia de Saussure, como la propuesta por Benjamin Hrushovski que Brian McHale utiliza en su libro Postmodernist Fiction:

(...) according to Benjamin Hrushovski all literary texts involve a "double-decker" structure of reference. Literary texts project at least one internal field of reference, a universe or semantic continuum (loosely, a "world") constructed in and by the text itself. In addition, they inevitably refer outside their internal field to an external field of reference: the objective world, the body of historical fact or scientific theory, an ideology or philosophy, other texts, and so on. (McHale 1987: 28-9) 
Para Hrushovski, toda pieza literaria lleva implícitos en mayor o menor medida un plano interno de referencia y otro externo; sin embargo, en términos saussurianos, la referencia interna no es referencia, sino sintaxis, gramática o coherencia lingüística.

Al margen de toda definición, Gass piensa que el texto es un dominio verbal que se justifica a sí mismo, por lo que no debe reflejarse en ninguna realidad ajena a él; el autor entiende que el mundo exterior sólo es un horizonte del lenguaje comunicativo, y que la literatura se debe en última instancia a esta otra realidad, al entramado o continuo semántico que el propio texto construye. Así se aprecia en el siguiente comentario de su libro de ensayos The World Within the Word:

(...) where the ancient dream of the rationalist -that somewhere in language there is a blueprint or a map of reality (where Eeyore's meadow's marked, and Piglet's tree, as well as where the Woozle wasn't) - that dream remains a dream because now language is the land -in fiction- where every fact has to have the structure of the sentence which states it, value too, and quality, and apprehension, since there is no out-of-doors in the world where language is the land. (Gass 1989: 316-7)

La paradoja intrínseca a la literatura declarada formalista se encuentra en el hecho de que entre sus objetivos se encuentra poner en práctica un distanciamiento verbal de la realidad, elaborando textos que difícilmente permiten una lectura mimética, pero que se erigen en artefactos verbales que aspiran a la integridad y coherencia de cualquier objeto real. Esto se acentúa en escritores que, como Gass, basan sus creaciones en estrategias metafóricas, inspirándose a menudo en la realidad para su elaboración. El ámbito de la referencia -o de la referencia externa en terminología de Hrushovski- no sólo no se llega a abandonar nunca, sino que es, de un modo abstracto, el destino último de estas creaciones.

El formalismo de los escritores autoreflexivos de segunda generación constituye en cierta medida un regreso a la modernidad: aunque puede estar determinado por intenciones disidentes, como se veía más arriba, es indiscutible que esta tendencia literaria tiene un marcado carácter estético que recupera valores como los de la totalidad y la autonomía poética característicos de las posiciones simbolistas más extremas. El propio autor se ha definido a sí mismo como un purified modernist es decir, un modernista renovado y depurado (Brooke K. Horvath, et al. 1983. "A Colloquy with William H. Gass”, 597). En realidad, las obras de muchos de estos autores constituyen lo que Sukenick describió como una escritura "más allá de la forma", una ficción que está en continuo proceso de definición; la forma no es un concepto o estructura estable, según este autor, sino que es "el contexto temporal de la imaginación”, una búsqueda literaria de la expresión asistemática y personal hasta la subversión (Sukenick 1985: 33).

El proyecto estético de William Gass está marcado por dualidades que a menudo parecen irreconciliables, y una de las que produce más confusión en la recepción de su obra se encuentra en su doble faceta como escritor de narrativa y como ensayista. Como él afirma en su libro de ensayos Finding a Form: "My stories are malevolently anti-narrative, and my essays are maliciously anti-expository" (Gass 1996: 46).

$\mathrm{Y}$, en efecto, sus novelas constituyen a menudo verdaderas teorías verbales, mientras que sus ensayos se aproximan con audacia al género poético. Esta práctica "mixta" es 
también frecuente en otros escritores de su generación, como el propio Sukenick -el autor con quien quizá Gass es más fácilmente comparable-, que lo explica afirmando que cuanto más se habla de una obra menos se participa en ella, por lo que asegura: "One of the tasks of modern fiction, therefore, is to displace, energize, and re-embody its criticism -to literally reunite it with our experience of the text" (Sukenick 1985: 5).

El siguiente comentario de Gass sugiere la apariencia problemática de su producción: "The Concepts of the philosopher speak, the words of the novelist are mute; the philosopher invites us to pass through his words to his subject: Man, God nature, moral law; while the novelist, if he is any good, will keep us kindly imprisoned in his language -there is literally nothing beyond" (Gass 1979: 8). Gass es ambas cosas: como filósofo, su misión consiste en disertar sobre su "asunto", que es principalmente el lenguaje; como escritor, su objetivo es rechazar el utilitarismo de la concepción más común del medio verbal y tratar de producir con él un efecto estético. Ésta es la clave de la dificultad con que se plantean las cuestiones de género en la obra de este autor, pues dicha dualidad aparentemente irreconciliable queda amalgamada en todos sus textos, independientemente de la apariencia bajo la que se presenten. Es posible que la obra de William Gass sea, de entre las de su generación, la que con mayor compromiso se ocupa de exhibir la tensión entre la afirmación y la negación de la significación: "In every art two contradictory impulses are in a state of Manichean war: the impulse to communicate and so to treat the medium of communication as a means, and the impulse to make an artifact out of the materials of the medium and so to treat the medium as an end" (Gass 1979: 94).

Los formalismos son, en general, difíciles de llevar a la práctica, quedando en su mayoría reducidos a atractivas teorías estéticas, y en literatura lo son todavía más, dados los condicionantes semánticos del medio verbal. Gass es consciente de ello, pero aun así no renuncia al juego de la creación absoluta; la diferencia radical de su proyecto con el de otros formalistas es que su intento de lograr la expresión artística suprema, que él concibe como un acontecimiento verbal metafórico íntegro y autosuficiente, pasa por una fase en que aparece reflejada esa realidad que otros evitan con recelo. Asumido el carácter elaborado e intuitivo de su empresa, la creación de una gran metáfora con cada una de sus obras se le antoja a Gass la estrategia perfecta que le permite idear obras metaficcionales como The Tunnel y Willie Masters'Lonesome Wife, piezas en las que el lenguaje aspira a alcanzar el ideal creativo del signo puro, aspiración que le acerca inevitablemente a Mallarmé.

\section{REFERENCIAS BIBLIOGRÁFICAS}

Bernstein, C. 1986. Content's Dream: Essays, 1975-1984. Los Angeles: Sun and Moon Press.

Brooke K. H, et al. 1983. "A Colloquy with William H. Gass”. Modern Fiction Studies 29:587-608

Duncan, J. L. 1976. “A Conversation with Stanley Elkin and William H. Gass”. Stanley Elkin and William H. Gass: A Special Feature. Iowa Review 7: 48-77.

Gass, W. 1979. Fiction and the Figures of Life. Boston: David R. Godine. 
. 1989. The World Within the Word. Boston: David R. Godine. . 1996. Finding a Form. New York: Alfred A. Knopf. . 2002. Tests of Time. New York: Alfred A. Knopf.

Hurtado Valero, P. M. 1994. Michel Foucault: Un proyecto de Ontología Histórica. Málaga: Ágora.

LeClair, T. y McCaffery, L., eds. 1982. Anything Can Happen: Interviews with Contemporary American Novelists. Urbana and London: University of Illinois Press.

McHale, B. 1987. Postmodernist Fiction. London and New York: Routledge.

Piqueras, B. 1995. "An Interview with William Gass”. San Luis (MO), USA. Sin publicar.

Sukenick, R. 1985. In Form: Digressions on the Act of Fiction. Carbondale and Edwardsville: Southern Illinois University Press.

Vernier, M. E. 1988. "Escritura lateral: Ensayos de Carlos Fuentes". La obra de Carlos Fuentes: Una visión múltiple. Ed. A. M. Hernández DE LóPez. Madrid: Pliegos. 331-337.

WebB, H. W. 1974. “The Singular Worlds of Jack Kerouac”. Contemporary American Novelists. Ed. H. T. Moore. Carbondale and Edwardsville: Southern Illinois University Press. 120-134. 